\title{
Magnetic Field Suppression of the Conducting Phase in Two Dimensions
}

\author{
D. Simonian, S. V. Kravchenko, and M. P. Sarachik \\ City College of the City University of New York, New York, NY 10031
}

(April 25, 2018)

\begin{abstract}
The anomalous conducting phase that has been shown to exist in zero field in dilute twodimensional electron systems in silicon MOSFETs is driven into a strongly insulating state by a magnetic field of about $20 \mathrm{kOe}$ applied parallel to the plane. The data suggest that in the limit of $T \rightarrow 0$ the conducting phase is suppressed by an arbitrarily weak magnetic field. We call attention to striking similarities to magnetic field-induced superconductor-insulator transitions.

PACS numbers: 71.30.+h, 73.40.Qv, and 74.76.Db
\end{abstract}

Recent experiments in high-mobility Si MOSFETs have provided strong evidence that a conducting phase exists in dilute two-dimensional (2D) electron systems in the absence of a magnetic field, in disagreement with predictions of the scaling theory [1] for non-interacting electrons. We attribute this finding to the availability of samples of unusually high mobility, allowing a transition from insulating to conducting behavior with increasing electron density, $n_{s}$, at small densities $\left(n_{s} \sim 10^{11} \mathrm{~cm}^{-2}\right)$. We note that since the Fermi energy $\epsilon_{F} \propto n_{s}$ in two dimensions and the electron correlation energy $\epsilon_{e e} \propto n_{s}^{1 / 2}$, the ratio $\epsilon_{e e} / \epsilon_{F}$ is proportional to $n_{s}^{-1 / 2}$; therefore, the lower the electron density, the greater the role of electronelectron interactions. For the 2D electron system in silicon, it has been shown experimentally [2] that the temperature $(T)$ and electric field $(E)$ dependences of the resistivity on the far-insulating side of the transition are consistent with the presence of a Coulomb gap in the density of states, indicating that electron correlations play a significant role. Moreover, comparison of temperature scaling and electric field scaling [3] near the $H=0$ transition in Si MOSFETs yields a dynamical exponent, $z \approx 0.8$, close to the value $z=1$ expected theoretically for a strongly interacting system (see, e.g., Ref. (4), again pointing to the importance of Coulomb interactions. Strong electron-electron interactions may thus be a central feature that allows the existence of a conducting phase in two dimensions. However, the nature of this phase remains unclear.

The influence of a magnetic field applied perpendicular to the plane of the 2D electron system has been studied in detail by Pudalov and coworkers [5] in highmobility MOSFETs with comparable electron densities. In these studies, the magnetoconductance is largely dominated by orbital effects which lead to the quantum Hall effect. In this Letter we report the results of measurements of the resistivity in a magnetic field applied parallel to the plane; here the magnetic field couples to the spins, but not to the orbital motion. Our results indicate that a parallel magnetic field has a dramatic effect on the transition, entirely eliminating the conduction mechanism responsible for the existence of the $H=0$ conducting phase above $\sim 20 \mathrm{kOe}$. Based on our data, we suggest that the conducting phase is suppressed by an arbitrarily weak magnetic field in the limit $T \rightarrow 0$. We point out further that the behavior in a magnetic field, as well as the critical behavior in zero field [3.6, , bear a strong resemblance to behavior reported near the superconductor-insulator transition in thin metal films [7,8,9, 9, raising the possibility that the anomalous conducting phase found in the $2 \mathrm{D}$ electron system in silicon MOSFETs is, in fact, a superconducting phase.

We report results of measurements of the linear and nonlinear DC resistivities of a high-mobility $\left(\mu_{T=4.2 K}^{\max } \approx\right.$ $24,000 \mathrm{~cm}^{2} / \mathrm{Vs}$ ) Si MOSFET sample; data for two other high-mobility samples gave similar results. We note that equivalent information is obtained from the temperaturedependence of the linear resistivity (in the limit $E \rightarrow 0$ ) and the electric-field-dependence of the (nonlinear) resistivity in the limit $T \rightarrow 0$, as was demonstrated [3] by similar behavior near the critical point in the two cases. Measurements as a function of electric field are easier to perform and entail smaller errors. As in earlier experiments, the electron density was set by adjusting the gate voltage. The resistivity was measured as a function of parallel magnetic field, at various temperatures, and for different values of the electric field (determined by the measuring current). No difference was found for in-plane magnetic fields applied parallel and perpendicular to the measuring current. The samples and measurements are described in more detail in Refs. [3:6].

Figure 1 shows the nonlinear resistivity in units of $h / e^{2}$ as a function of electric field in a magnetic field of $5 \mathrm{kOe}$ at a temperature of $0.1 \mathrm{~K}$. Each curve corresponds to a different electron density (gate voltage). For comparison, the inset shows the resistivity as a function of electric field in the absence of a magnetic field for comparable electron densities. In zero magnetic field, the curves clearly separate into two groups: for low electron densities the resistivity increases with decreasing temperature (insulating behavior), while for higher electron densities the resistivity decreases with decreasing temperature (conducting behavior); the resistivity at the transition $\left(n_{s}=n_{c}\right)$ is independent of electric field and approximately equal 


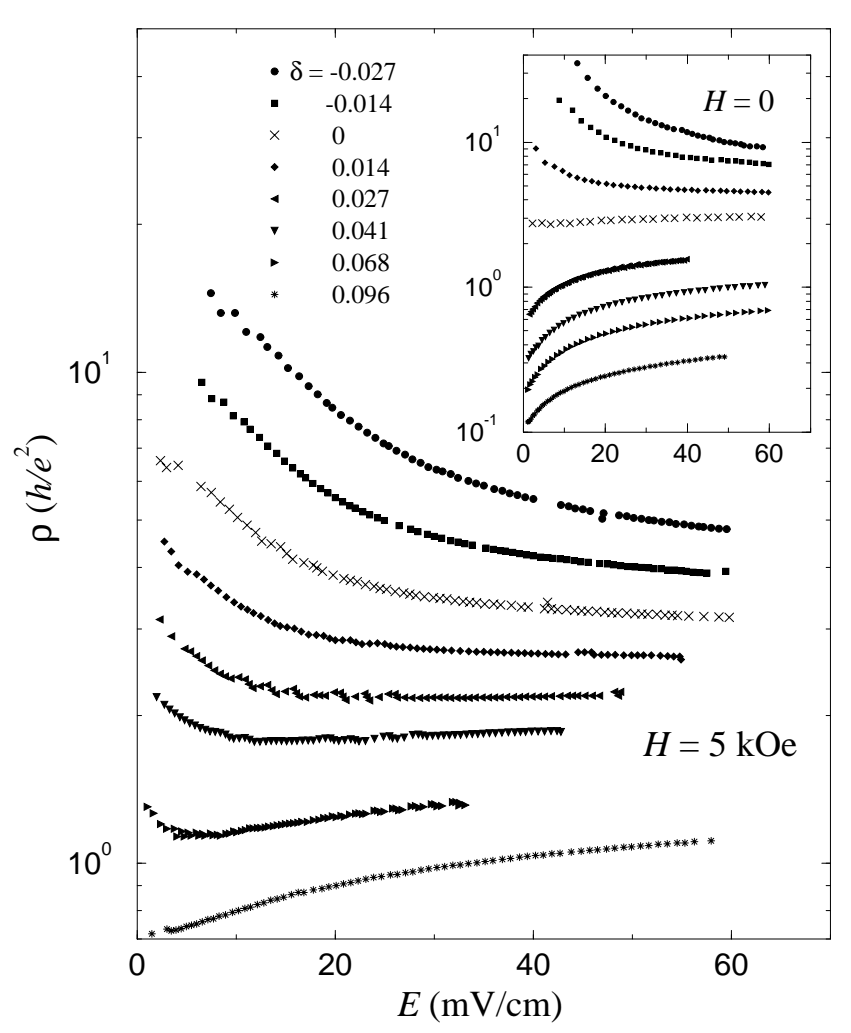

FIG. 1. Resistivity as a function of electric field on a semilogarithmic scale at $H_{\|}=5 \mathrm{kOe}$ and $T=0.10 \mathrm{~K}$. Electron densities are specified relative to the $H=0$ critical density, $n_{c}=8.03 \times 10^{10} \mathrm{~cm}^{-2} ; \delta \equiv\left(n_{s}-n_{c}\right) / n_{c}$. The inset shows $\rho(E)$ in the absence of a magnetic field at $T=0.22 \mathrm{~K}$, for $\delta=-0.065,-0.050,-0.030,0,0.052,0.10,0.16$, and 0.27 . The crosses correspond to $\delta=0$.

to $3 h / e^{2}$. As demonstrated in reference [3], a single (horizontal) multiplicative factor can be used to obtain scaling. The effect of a parallel magnetic field is clearly shown in the main part of Fig. 1: a magnetic field of $5 \mathrm{kOe}$ drives all curves toward more insulating behavior. Moreover, there is a qualitative change: for some electron densities the resistivity exhibits non-monotonic behavior, developing a shallow minimum. We shall return to this point below.

The resistivity is shown on a logarithmic scale as a function of magnetic field at a fixed temperature of $0.25 \mathrm{~K}$ in Fig. 2 for three different electron densities on the conducting side of the $H=0$ transition $\left(n_{s}>n_{c}\right)$. The resistivity initially stays approximately constant up to $H_{\|} \approx 4 \mathrm{kOe}$; data at low fields are shown on an expanded scale in the inset to Fig. 1 for an electron density corresponding to $\delta \equiv\left(n_{s}-n_{c}\right) / n_{c}=0.15$. The resistivity then increases sharply as the magnetic field is raised further, changing by almost three orders of magnitude. Above $H_{||} \sim 20 \mathrm{kOe}$, it saturates and stays approximately constant up to the highest measured field, $H_{\|}=70 \mathrm{kOe}$. A parallel magnetic field has dramatically altered the

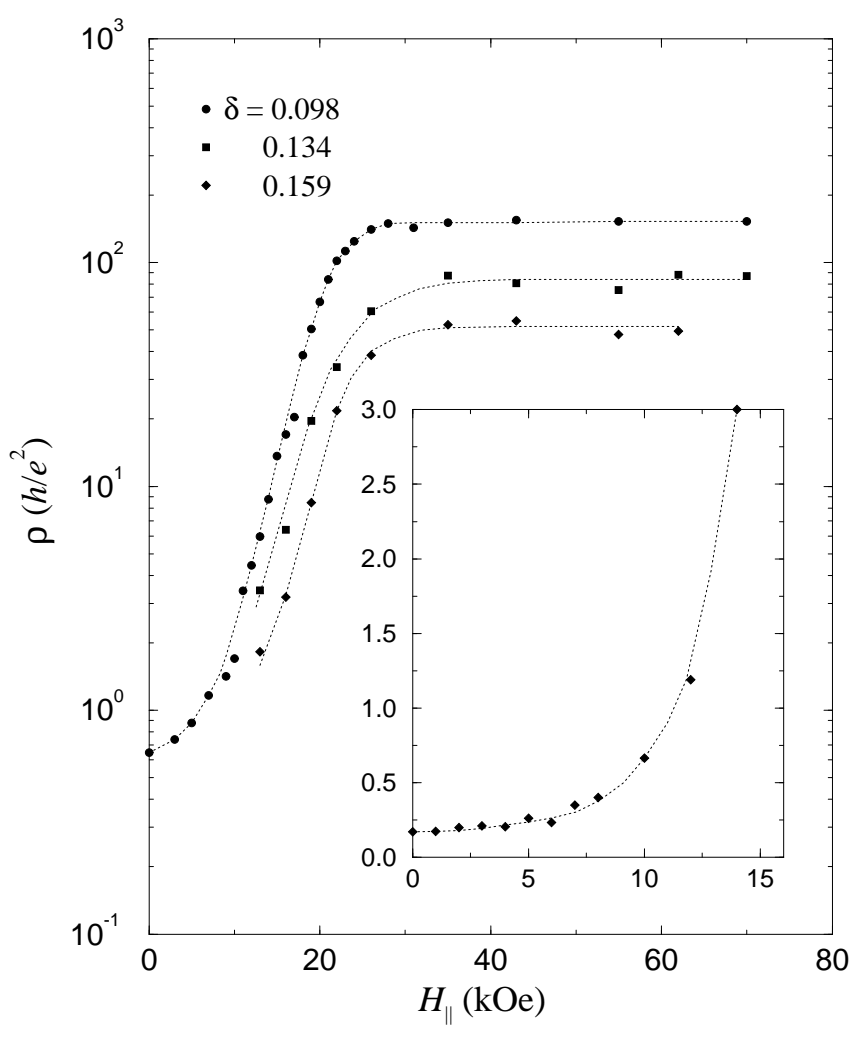

FIG. 2. Resistivity on a logarithmic scale as a function of a magnetic field applied parallel to the plane at $T=0.25 \mathrm{~K}$ for three electron densities. The inset shows the resistivity on a linear scale in small magnetic field for $\delta=0.15$ and $T=0.13 \mathrm{~K}$.

system, apparently suppressing the conduction mechanism in the anomalous conducting phase entirely in fields above $20 \mathrm{kOe}$. The behavior is strongly reminiscent of the quenching of superconductivity by a magnetic field (except, of course, that the zero-field resistivity in our case is finite rather than zero). The Zeeman energy, $g \mu_{B} H_{\|}$, at $20 \mathrm{kOe}$ corresponds to a thermal energy $k_{B} T_{H}$ with $T_{H}=2.7 \mathrm{~K}$. Note that $T_{H} \sim T^{*} \approx 2 \mathrm{~K}$ where $T^{*}$ marks the onset of the low-temperature conducting phase in zero field (see the lowest curve of Fig. 价).

Measurements in magnetic fields oriented perpendicular to the plane of the electrons confirm earlier detailed magnetotransport results obtained by Pudalov et al [5] in Si MOSFETs with comparable electron densities and mobilities. Fig. 1 of their paper shows that the resistance is essentially constant up to $5 \mathrm{kOe}$, above which it rises sharply before it is overwhelmed by the quantum Hall effect above $\sim 15 \mathrm{kOe}$. This puzzling, sharp initial increase has been the subject of some debate. We suggest that its origin is the same as for a parallel field: a conducting phase exists at low temperatures which is suppressed by a magnetic field. Thus, the anomalous $H=0$ conducting state is driven into a strongly insulating ("normal") state either by $H_{\|}$or by $H_{\perp}$, in a qualitatively similar way.

We now consider whether one can identify a critical 


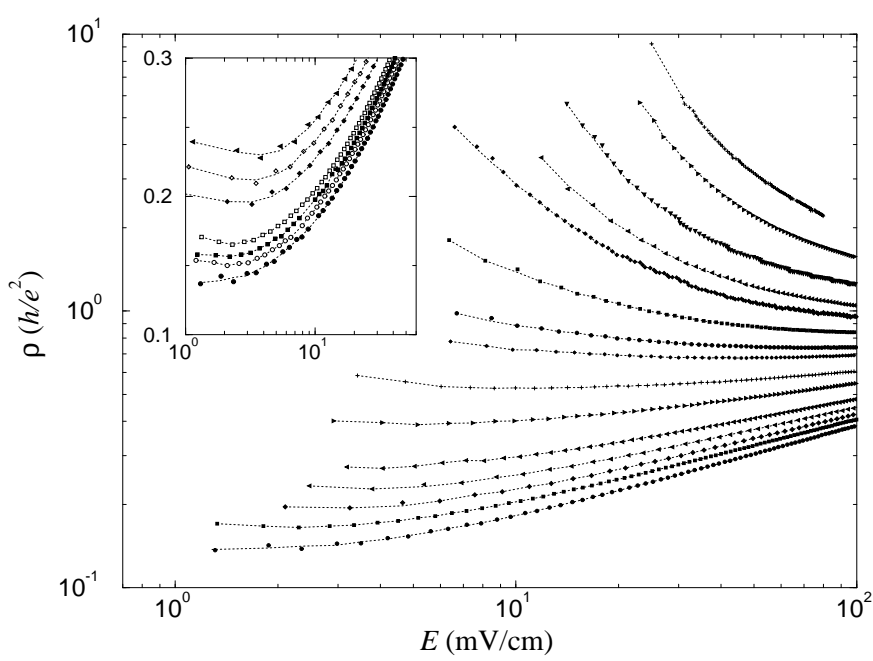

FIG. 3. Isomagnetic curves of nonlinear resistivity as a function of electric field on a log-log scale for fixed electron density, $\delta=0.3$, at $T=0.10 \mathrm{~K}$. Each curve corresponds to a different value of parallel magnetic field, $H_{\|}=0$ (bottom curve), 6, 8, 10, 12, $15,17,19,20,22,24,25,27,30$, and 34 kOe. Minima in the resistivity are clearly illustrated in the inset, where data are shown on a linear scale for $H_{\|}=0$ (bottom curve), 4, 5, 6, 8, 9, and $10 \mathrm{kOe}$

parallel magnetic field below which the system is a conductor, and above which it is an insulator. In Fig. 3, we plot the nonlinear resistivity, $\rho(E)$, for a fixed electron density (corresponding to a zero-field $\delta=0.3$ ) at $0.1 \mathrm{~K}$. Here each curve corresponds to a different value of $H_{\|}$. As noted above, the curves are qualitatively different from those in zero field shown in the inset to Fig. 1: the curves for $\delta>0$ display a shallow minimum in finite magnetic field, and it is no longer possible to use a single parameter to collapse them onto two separate branches, insulating and conducting, as was done at $H=0$ [3.6. Moreover, there is no universal "critical" value of the resistivity, $\rho\left(H_{\| c}\right)$. This suggests that any finite magnetic field (at $T=0)$ drives the system into the insulating phase.

Finally, Fig. 1 shows the linear resistivity (at $E \rightarrow 0$ ) as a function of temperature for a fixed electron density on the conducting side of the $H=0$ transition $(\delta=0.1)$ in several parallel magnetic fields between 0 and $14 \mathrm{kOe}$. The zero-field curve is typical of a conductor, with resistance dropping sharply as the temperature is decreased below $\approx 2 \mathrm{~K}$, while at $H=14 \mathrm{kOe}$ it is strongly insulating. Note that the magnetic field has almost no effect on the resistivity above $T^{*} \approx 2 \mathrm{~K}$, while below $T^{*}$ the effect of $H_{\|}^{*}$ is enormous (as discussed earlier, $T^{*}$ is the characteristic temperature below which the conducting phase exists in zero field). We note the presence of resistivity minima at intermediate magnetic fields. Again, one-parameter scaling with temperature breaks down, as did one-parameter scaling with electric field (see above).

The effect of a parallel magnetic field in the 2D

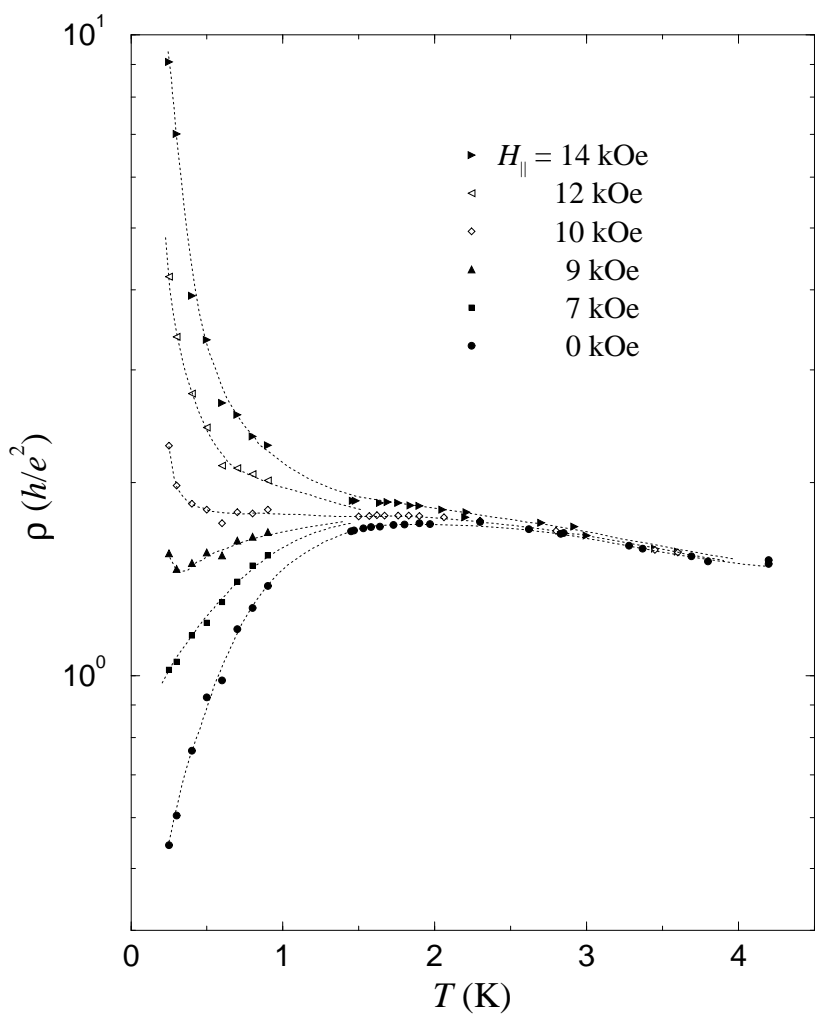

FIG. 4. Linear resistivity versus temperature in five different parallel magnetic fields. The electron density corresponds to $\delta=0.10$.

electron gas in Si MOSFETs strongly resembles the effect of a field in systems that undergo magnetic field-induced superconductor-to-insulator transitions (SIT). For example, the isomagnetic curves of Fig. A are similar to those in Fig. 1 of Ref. [7] measured near an SIT driven by a magnetic field in disordered indium oxide films. They are similar also to the temperature dependence of the resistivity of a bulk high- $T_{c}$ compound [10].

The possibility of superconductivity in Si MOSFETs has been considered by Takada 111 and by Hanke and Kelly [12]. More recently, $p$-wave superconductivity in this system has been proposed by Phillips and Wan [13]. In addition, various kinds of instabilities in 2D have been proposed theoretically (for review, see Ref. [14]), including Wigner crystallization, a transition to a ferromagnetic state at low electron densities, single-valley occupancy, and instabilities toward a charge-density or spindensity ground state.

To summarize, we report that a parallel magnetic field suppresses the anomalous conducting phase found at $H=0$ in the $2 \mathrm{D}$ electron system in Si MOSFETs. The resistivity increases by several orders of magnitude at low temperatures, saturating above $\approx 20 \mathrm{kOe}$. Qualitatively similar behavior is found [5] in perpendicular field, which couples to orbital motion as well as spin, up to approximately $15 \mathrm{kOe}$; at higher perpendicular fields the magnetoconductance is overwhelmed by the quantum 
Hall effect. The fact that a parallel magnetic field has such a dramatic effect indicates that the electrons' spins play a central role. The fact that the Zeeman energy $g \mu_{B} H$ and thermal energy $k_{B} T$ that destroy the conducting phase are roughly comparable further supports this possibility. One-parameter scaling with temperature and electric field, found to hold when $H=0$, breaks down even in a weak magnetic field, suggesting the elimination of the conducting phase by an arbitrarily small $H$. The magnetoresistance strongly resembles that observed at the superconductor-to-insulator transition driven by a magnetic field.

We thank R. N. Bhatt, V. Dobrosavljević, A. B. Fowler, P. Phillips, S. Sachdev, and S. L. Sondhi for valuable discussions and Sera Cremonini for help in the analysis of the experimental data. This work was supported by the US Department of Energy under Grant No. DE-FG0284ER45153.

[1] E. Abrahams, P. W. Anderson, D. C. Licciardello, and T. V. Ramakrishnan, Phys. Rev. Lett. 42, 673 (1979).

[2] W. Mason, S. V. Kravchenko, and J. E. Furneaux, Surf. Sci. 361/362, 953 (1996).
[3] S. V. Kravchenko, D. Simonian, M. P. Sarachik, W. Mason, and J. E. Furneaux, Phys. Rev. Lett. 77, 4938 (1996).

[4] S. L. Sondhi, S. M. Girvin, J. P. Carini, and D. Shahar, Rev. Mod. Phys. 69, 315 (1997).

[5] See, e.g., V. M. Pudalov, M. D'Iorio, and J. W. Campbell, Surf. Sci. 305, 107 (1994).

[6] S. V. Kravchenko, G. V. Kravchenko, J. E. Furneaux, V. M. Pudalov, and M. D'Iorio, Phys. Rev. B 50, 8039 (1994); S. V. Kravchenko, W. E. Mason, G. E. Bowker, J. E. Furneaux, V. M. Pudalov, and M. D'Iorio, Phys. Rev. B 51, 7038 (1995).

[7] A. F. Hebard and M. A. Paalanen, Phys. Rev. Lett. 65, 927 (1990).

[8] Y. Liu, K. A. McGreer, B. Nease, D. B. Haviland, G. Martinez, J. W. Halley, and A. M. Goldman, Phys. Rev. Lett. 67, 2068 (1991).

[9] A. Yazdani and A. Kapitulnik, Phys. Rev. Lett. 74, 3037 (1995).

[10] Y. Ando, G. S. Boebinger, A. Passner, T. Kimura, K. Kishio, Phys. Rev. Lett. 75, 4662 (1995); K. Karpińska et al., Phys. Rev. Lett. 77, 3033 (1996).

[11] Y. Takada, J. Phys. Soc. Japan 45, 786 (1978); J. Phys. Soc. Japan 49, 1713 (1980).

[12] W. Hanke and M. J. Kelly, Phys. Rev. Lett. 45, 1203 (1980); M. J. Kelly and W. Hanke, Phys. Rev. B 23, 112 (1981).

[13] P. Phillips and Y. Wan, submitted to Phys. Rev. Lett.

[14] T. Ando, A. B. Fowler, and F. Stern, Rev. Mod. Phys. 54, 437 (1982). 